\title{
Influence of the Bilayer Thickness of Nanostructured Multilayer MoN/CrN Coating on Its Microstructure, Hardness, and Elemental Composition
}

\author{
A. D. Pogrebnyak ${ }^{a, *}$, O. V. Bondar ${ }^{a, b}$, B. Zhollybekov, S. Konstantinov ${ }^{d}$, P. Konarski ${ }^{e}$, \\ V. M. Beresnev ${ }^{f}$, and A. I. Kupchishin ${ }^{g}$ \\ a Sumy State University, Sumy, Ukraine \\ ${ }^{b}$ NanoBioMedical Centre, Adam Mickievicz University, Poznan, Poland \\ ${ }^{c}$ Berdakhay Karakalpak State University, Nukus, Uzbekistan \\ ${ }^{d}$ Belarusian State University, Minsk, Republic of Belarus \\ e Tele and Radio Research Institute, Warsaw, Poland \\ ${ }^{f}$ Karazin Kharkiv National University, Kharkiv, Ukraine \\ ${ }^{g}$ Abai Kazakh National Pedagogical University, Almaty, Kazakhstan \\ *e-mail:alexp@i.ua \\ Received April 28, 2016; in final form, March 14, 2017
}

\begin{abstract}
Multilayer nanostructured coatings consisting of alternating MoN and CrN layers were obtained by vacuum cathode evaporation under various conditions of deposition. The transition from micron sizes of bilayers to the nanometer scale in the coatings under investigation leads to an increase in hardness from 15 to $35.5 \mathrm{GPa}$ (with a layer thickness of about $35 \mathrm{~nm}$ ). At the same time, when the number of bilayers in the coating decreases, the average Vickers hardness increases from $1267 \mathrm{HV}_{0.05}$ to $3307 \mathrm{HV}_{0.05}$. An increase in the value of the potential supplied to the substrate from -20 to $-150 \mathrm{~V}$ leads to the formation of growth textures in coating layers with the [100] axis, and to an increase in the intensity of reflections with increasing bilayer thickness. Elemental analysis carried out with the help of Rutherford backscattering, secondary ion mass spectrometry and energy dispersion spectra showed a good separation of the MoN and CrN layers near the surface of the coatings.
\end{abstract}

DOI: $10.1134 / \mathrm{S} 1063783417090232$

\section{INTRODUCTION}

Vacuum-arc coatings on the basis of transition metal nitrides have high hardness and good adhesion to the substrate [1-4]. Upon the transition of nitride coatings to the nanostructured state there is a significant improvement in their physicomechanical and tribological properties [5], an increase in resistance to thermal impacts [6-9], improvement of corrosion resistance [10-14], etc. One method to realize the transition of coatings to the nanostructured state is the deposition of multilayer composite materials [15-17], in which the alternation of layers from different components [14, 18] serves as a good diffusion barrier [19] and provides an increase in stability under external influences [20]. In this case, the superhard state of multilayer nanocomposites supposes the suppression of growth processes of germinating cracks, generation and propagation of dislocations, which provide the release of plastic deformation at a decrease in the size of nanocrystallites to $10 \mathrm{~nm}$ [21], and the absence of the anomalous Hall-Petch dependence inherent to the nanostructured materials [22].

As one of the most promising multilayer systems, the systems based on refractory metal nitrides or carbides, such as MoN/CrN [22], have been recently examined, since these compositions achieve high hardness and wear resistance of coatings in combination with high resistance to oxidation and other impacts of aggressive environment [23].

This work is devoted to studying the influence of the thickness of bilayers and deposition parameters on the phase composition, microstructure, and hardness of coatings deposited at different negative bias potentials $U_{b}$ supplied to the substrate, which provide an increase in the energy of deposited particles.

\section{METODS OF COATINGS FABRICATION AND INVESTIGATION}

Coatings were deposited on steel substrates by means of cathodic vacuum-arc evaporation in a Bulat 
6-M setup [20]. The pressure of the working atmosphere (nitrogen) in the chamber was practically constant $\left(P_{\mathrm{N}}=3 \times 10^{-3}\right.$ Torr $)$; the deposition rate was about $3 \mathrm{~nm} / \mathrm{s}$. The deposition was carried out using two targets: molybdenum and chromium cathodes-at a fixed holding time and continuous rotation of the fixed samples with a rate of $8 \mathrm{rpm}$. The total thickness of the coatings was approximately $10 \mu \mathrm{m}$ the deposition of coatings, a permanent negative potential $U_{b}=$ -20 and $-150 \mathrm{~V}$ was fed on a stainless steel substrate. Structural phase analysis was carried out by X-ray diffractometry (XRD) using $\mathrm{Cu} K_{\alpha}$ radiation at a X'Pert PANanalytical setup with a step of $0.05^{\circ}$ (at angles in the range $2 \theta=30^{\circ}-80^{\circ}$ ). Separation of profiles into constituent fragments was carried out using Powder Cell software. Elemental analysis and investigation of the cross-sections of coatings were carried out by several methods, namely by scanning electron microscopy (SEM) and microanalysis using a JEM-7001 TTLS (JEOL) microscope in the SEI and COMPO modes. Samples with coatings were cut with a diamond disc using a Buehler IsoMet Lowspeed Saw device, then a part of the coating on the cut was etched by a focused beam of gallium ions using a JEOL JIB4000 Focused Ion Beam System. To study the depth distribution profiles of elements of the coating, a $1.4 \mathrm{MeV} \mathrm{He}^{+}$ion beam with an energy resolution of $16 \mathrm{keV}$ was used in the geometry of Rutherford backscattering (RBS). To calculate the profiles of elements in the coating, a RESOLNRA software was used.

For elemental analysis, the method of secondary ion mass spectroscopy (SIMS) was also used. The studies were carried out on a SAJW 05 setup equipped with a Physical Electronics gun of argon ions and a QMA 410 Balzers quadrupole mass analyzer. Before the beginning of the research, preliminary mass spectra were measured in order to calibrate the setup. The dimensions of the etched crater were $2.5 \times 2.5 \mathrm{~mm}$, the ion beam energy was set equal to $1.72 \mathrm{keV}$, the current $-0.3 \times 10^{-6} \mathrm{~A}$, and the average sputtering rate was about $0.65 \mathrm{~nm} / \mathrm{min}$. Secondary ions were recorded only in the central part of the crater, the area of which was only $15 \%$ of the total area. The fairly low energies of the beam made it possible to eliminate the effect of ion mixing that occurs during sputtering of the coating material.

Hardness measurements were performed using a DM-8 instrument with Vickers pyramid, and a load of 500 and $100 \mathrm{~g}$.

\section{RESULTS AND DISCUSSION}

Energy-dispersive analysis of elemental composition of coatings showed that for coatings of all series, obtained both at low $\left(U_{b}=-20 \mathrm{~V}\right)$ and high voltage $\left(U_{b}=-150 \mathrm{~V}\right)$, the composition of metal components is close to the equiatomic one, and the atomic $\mathrm{Mo} / \mathrm{Cr}$ ratio varies from 0.90 to 0.93 . Consequently, it can be
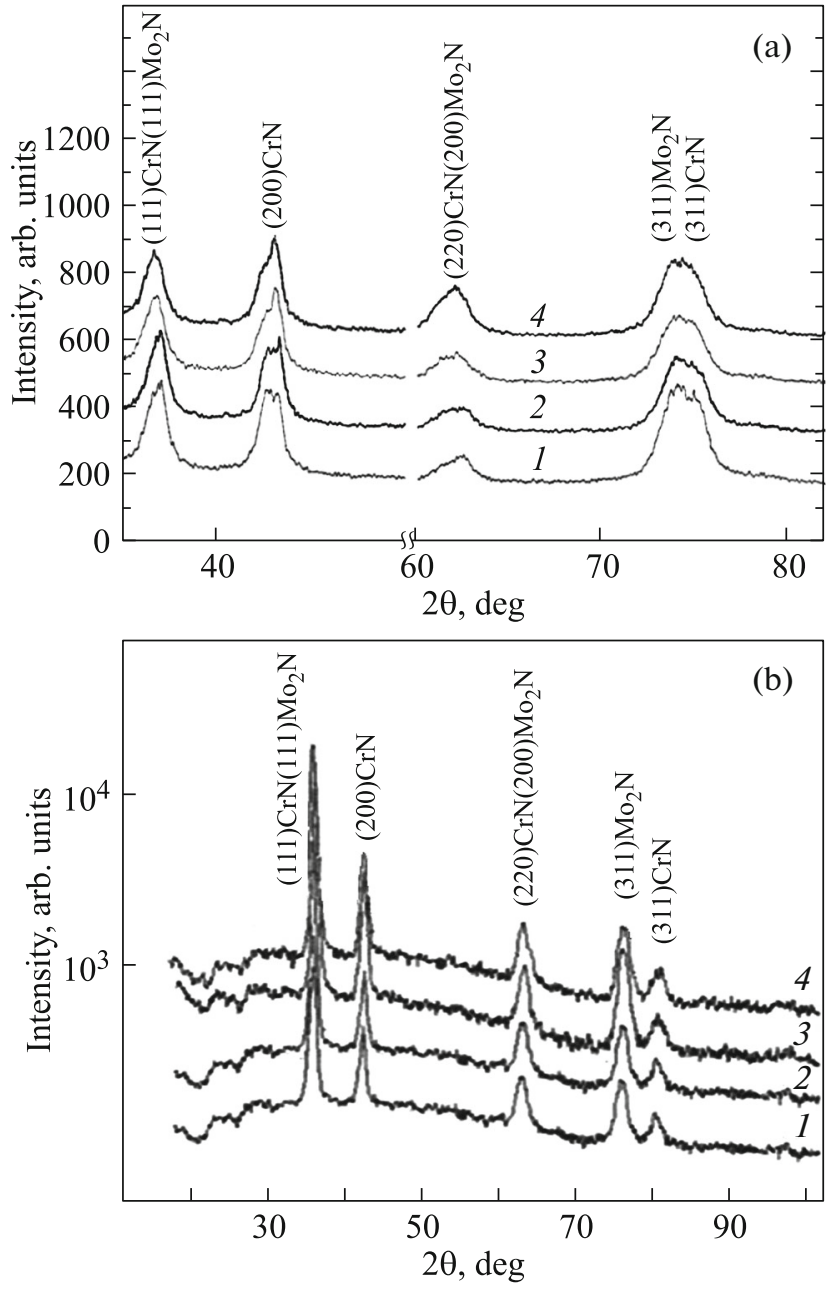

Fig. 1. Domains of diffraction spectra of coatings obtained at voltages $U_{b}=-20$ (a) and $-150 \mathrm{~V}$ (b) and layer thicknesses $h=300$ (1), 70 (2), 20 (3), and $12 \mathrm{~nm}$ (4).

argued that the thicknesses of resulting layers will be almost equal. With a change in $U_{b}$ from -20 to $-150 \mathrm{~V}$, the concentration of nitrogen atoms remains practically unchanged. XRD studies show that the value of $U_{b}$ significantly influences the forming structural state. From the comparison of Figs. 1a, 1b, we can see that, for a small potential $U_{b}=-20 \mathrm{~V}$, the forming crystalline lattices have the same type: crystal lattices of structural type $\mathrm{B} 1$ ( $\mathrm{fcc} \mathrm{NaCl}$ ), which is characteristic for $\mathrm{CrN}$ and $\gamma-\mathrm{Mo}_{2} \mathrm{~N}$. In the course of investigation, the predominant orientation of crystallite growth with the axis [311] perpendicular to the growth plane was observed, which is manifested in the relative intensification of the corresponding reflex. However, this type of texture is more pronounced in $\mathrm{CrN}$ layers. Setting a higher bias potential $U_{b}=-150 \mathrm{~V}$ leads to the different type of texture-[111], which improves with increasing layer thickness, but does not lead to an explicit separation of reflections from the corresponding phases of the two nitride layers at large diffraction 


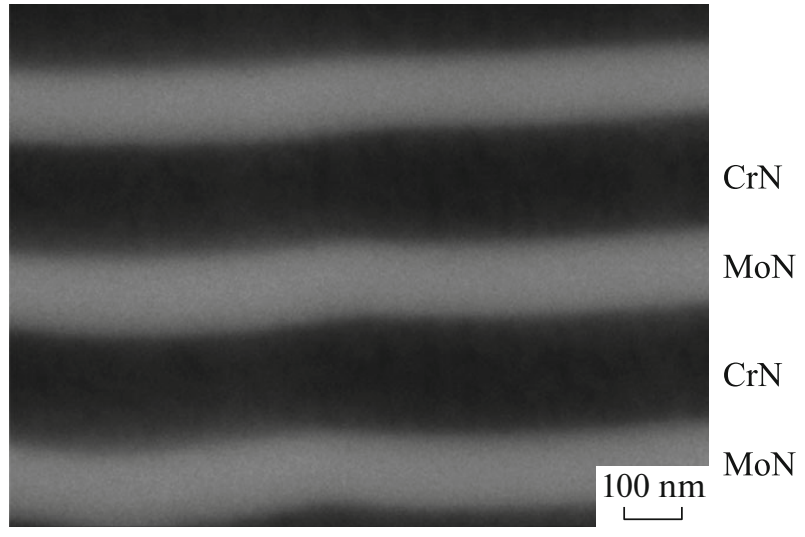

Fig. 2. SEM image of the lateral section of the coating corresponding to diffraction spectrum 1 in Fig. 1b, obtained with a magnification of 100000 times.

angles, which indicates the formation of a solid solution.

When potential $U_{b}=-150 \mathrm{~V}$ is supplied, a polycrystalline nontextured state grows. In the deposited layers, $\mathrm{CrN}$ and $\gamma-\mathrm{Mo}_{2} \mathrm{~N}$ mononitrides are formed with a cubic lattice of the $\mathrm{NaCl}$ type. The formation of this type texture is due to a relative decrease in the nitrogen concentration in the coating with an increase in the magnitude of the bias potential applied to the substrate. The presence of structures with the same interplanar distances in the contacting layers indicates an interrelated growth of these two structures.

At the substructural level, the size of nanocrystallites in the case of deposition of nanoscale layers is $L=12 \mathrm{~nm}$, and microdeformations of crystallites are $\langle\varepsilon\rangle=0.4-0.5 \%$. The obtained values of microdeformation are somewhat higher in coatings deposited when a low bias potential is supplied to the substrate than in coatings obtained by feeding higher bias potentials to the substrate. This fact is explained by the lower mobility of the deposited atoms, leading to a decrease in the diffusion healing probability of growth defects. Separation of reflections from the corresponding phases of the two layers is not observed, which additionally indicates the formation of a solid solution.

The SEM image of the cross section of the multilayer coating (44 layers, the total thickness of the coating is on the order of $7 \mu \mathrm{m}$ ) is shown in Fig. 2. The layers are well resolved using a scanning electron microscope and have clearly discernible boundaries.

Samples with MoN/CrN coating (180 layers) were studied using Rutherford backscattering of $\mathrm{He}^{+}$ions. It was found that the beam permeates eleven bilayers. The thicknesses of $\mathrm{CrN}$ and MoN layers in all periods are 86 and $75 \mathrm{~nm}$, respectively, which gives a bilayer thickness of $151 \mathrm{~nm}$. Figure 3 shows the depth distribution profiles of $\mathrm{Cr}$, Mo, $\mathrm{N}$ elements (up to a depth of $1800 \mathrm{~nm}$ ), which do not mix during deposition, at least up to the depth of the analysis of the ion beam.

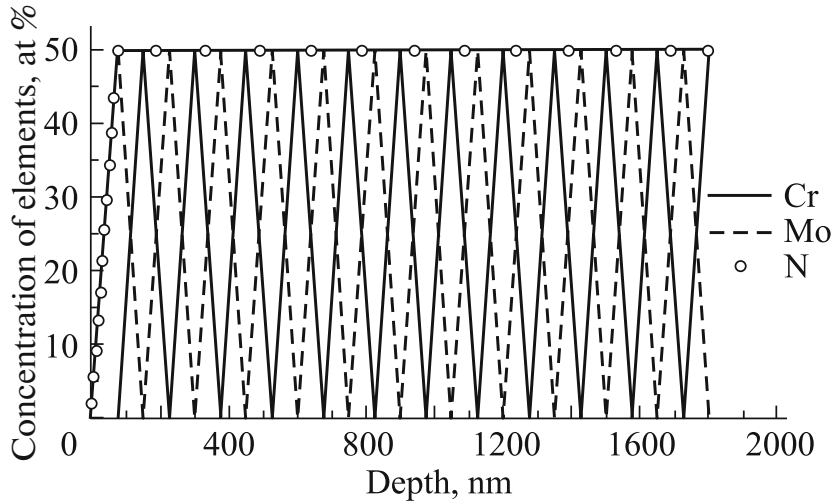

Fig. 3. Depth distribution profiles of elements in the coatings obtained using RBS spectra of $\mathrm{He}^{+}$ions on samples with MoN/CrN coatings (180 layers).

At the same time, the results of SIMS studies presented in Fig. 4 supplement the RBS data and also indicate the good quality of the deposited coatings. Due to the different sputtering rates of $\mathrm{CrN}$ and $\mathrm{MoN}$ layers, a somewhat lower thickness of the layers is observed. Therefore, by normalizing the SIMS results to the results of the RBS analysis, namely to the thickness of the layers obtained by the RBS method with an accuracy of $0.1 \mathrm{~nm}$, we obtain more real values of the nitrogen concentration profiles in the investigated layers from the SIMS results. From the profiles of elements shown on Fig. 4, it is clearly seen that the layers of chromium nitride have a higher nitrogen concentration than the nitride layers of molybdenum, as evidenced by an increase in the intensity of nitrogen peaks that coincide with the positions of chromium peaks. The results are in good agreement with XRD studies. As follows from the results of studying hardness of such coatings (Fig. 5), at a low voltage $U_{b}=$ $-20 \mathrm{~V}$, when the thickness of layers decreases, the hardness of the multilayer composite increases. This may be due to the characteristic effects of grain size reduction (the Hall-Petch equation) [22]. When a higher bias potential $U_{b}=-150 \mathrm{~V}$ is fed, such a trend takes place up to layer thicknesses of about $40 \mathrm{~nm}$, while a lower hardness is observed at lower thicknesses, which can be explained by the large number of boundaries and an increase in their volume fraction in the coating. The reason for this drop is the loss of the barrier properties of the phase boundary due to the formation of a solid solution (Mo, Cr) $\mathrm{N}$ caused by the mixing of high-energy particles (at a bias potential $U_{b}=-150 \mathrm{~V}$ ) at the boundaries of individual layers. The maximum hardness of coatings is achieved when the $\mathrm{CrN}$ phase having a cubic lattice and a relatively small region of homogeneity of the composition is present in $\mathrm{Cr}-\mathrm{N}$ layers. The maximum hardness (up to $35.5 \mathrm{GPa}$ ) was observed in coatings in which the $\mathrm{Mo} / \mathrm{Cr}$ ratio is 0.9 . 


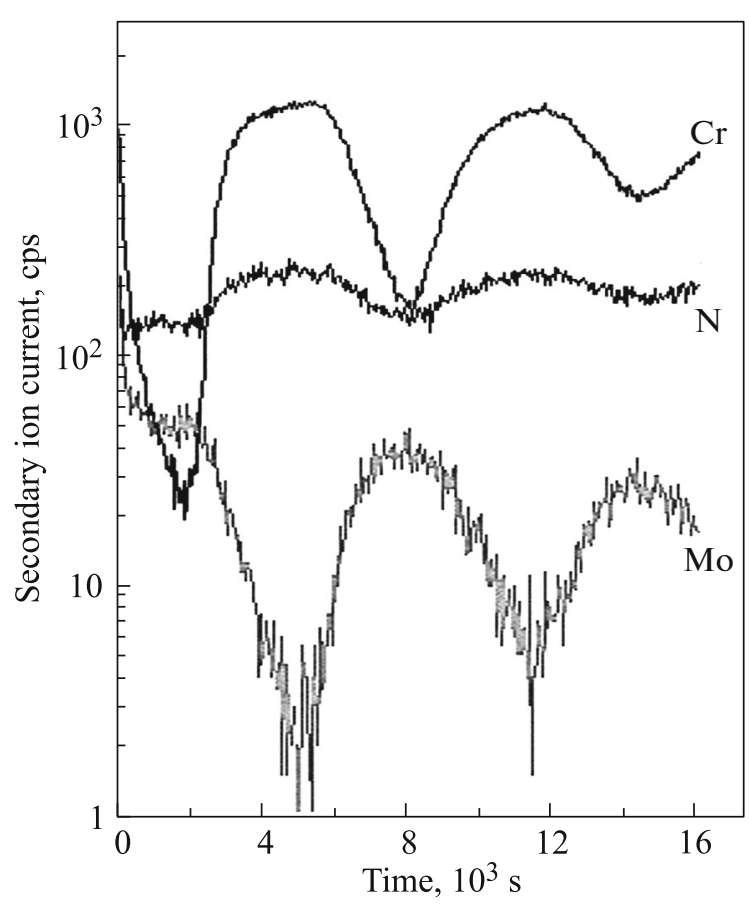

Fig. 4. The results of SIMS studies of $\mathrm{MoN} / \mathrm{CrN}$ coating (180 layers): the first four layers on an enlarged scale.

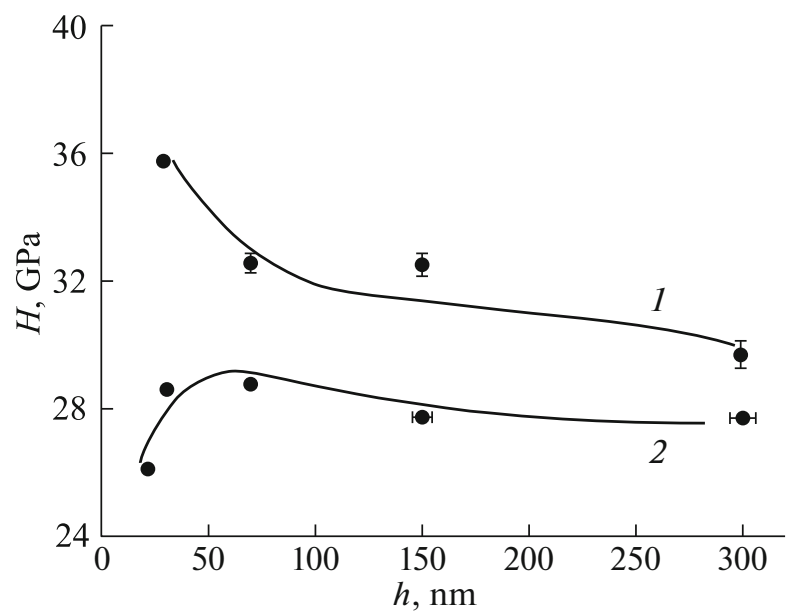

Fig. 5. Microhardness $H$ as a function of the thickness $h$ of the layer for coatings obtained at $U_{b}=-20(1)$ and $-150 \mathrm{~V}$ (2). Measurement errors are indicated as confidential Intervals.

\section{CONCLUSIONS}

The phase and elemental analysis of micro- and nanostructured multilayer coatings based on $\mathrm{MoN} / \mathrm{CrN}$ has been performed, as well as the study of their hardness depending on different deposition regimes. As a result of a change in the bias potential supplied to the substrate (from -20 to $-150 \mathrm{~V}$ ), as well as an increase in the thickness of bilayers in the coatings from 24 to $300 \mathrm{~nm}$, different structures were obtained: from cubic (such as $\mathrm{NaCl}$ ) to the formation of a solid solution. Such deposition conditions significantly affect the change in the growth structure of multilayer coatings. When obtaining $\mathrm{CrN}$ and $\gamma-\mathrm{Mo}_{2} \mathrm{~N}$ phases of coatings with a cubic lattice, a maximum hardness of $35.5 \mathrm{GPa}$ was observed. The coincidence of interplanar distances in contacting layers of chromium and molybdenum nitrides indicates the interconnected growth of these two structures.

\section{ACNOWLEDGMENTS}

The authors thank F.F. Komarov (Belarusian State University, Minsk) for his assistance in the measurements of the RBS spectra; B. Peplinska and E. Coy (NanoBioMedical Centre, Adam Mickiewicz University) for their assistance in SEM measurements of the structure of coatings, and O.V. Sobol' for his help in the interpretation of the XRD spectra.

The work was carried out within the framework of budgetary tasks no. 0115U000682 "Development of material science basics of structural engineering of vacuum-plasma superhard coatings in order to achieve the necessary functional properties" and no. 0116U006816 "Development of promising nanostructured multilayer coatings with improved physicomechanical and tribological properties".

\section{REFERENCES}

1. Nanostructured Coatings, Ed. by A. Cavaleiro and J. T. M. de Hosson (Springer, New York, 2006).

2. R. L. Boxman, V. N. Zhitomirsky, I. Grimberg, L. Rapoport, S. Goldsmith, and B. Z. Weiss, Surf. Coat. Technol. 125, 257 (2000).

3. P. H. Mayrhofer, C. Mitterer, L. Hultman, and H. Clemens, Prog. Mater. Sci. 51, 1032 (2006).

4. A. D. Pogrebnyak, A. P. Shpak, N. A. Azarenkov, and V. M. Beresnev, Phys. Usp. 52, 29 (2009).

5. A. D. Pogrebnjak, A. G. Ponomarev, A. P. Shpak, and Yu. A. Kunitskii, Phys. Usp. 55, 270 (2012).

6. T. N. Koltunowicz, P. Zukowski, V. Bondariev, K. Czarnacka, O. Boiko, J. A. Fedotova, and J. V. Kasiuk, J. Alloys Compd. 650, 262 (2015).

7. Ya. A. Lyashenko and A. N. Zaskoka, Tech. Phys. 60, 1014 (2015).

8. S. N. Danilchenko, A. V. Koropov, I. Y. Protsenko, B. Sulkio-Cleff, and L. F. Sukhodub, Cryst. Res. Technol. 41, 268 (2006).

9. T. N. Koltunowicz, P. Zhukowski, V. Bondariev, J. A. Fedotova, and A. K. Fedotov, Acta Phys. Polon. A 123, 932 (2013).

10. V. M. Beresnev, O. V. Sobol', A. D. Pogrebnjak, P. V. Turbin, and S. V. Litovchenko, Tech. Phys. 55, 871 (2010).

11. M. K. Samani, X. Z. Ding, N. Khosravian, B. AminAhmadi, Y. Yi, G. Chen, E. C. Neyts, A. Bogaerts, and B. K. Tay, Thin Solid Films 578, 133 (2015). 
12. Z. H. Xie, M. Hoffman, P. Munroe, R. Singh, A. Bendavid, and P. J. Martin, J. Mater. Res. 22, 2312 (2007).

13. J. Lackner, L. Major, and M. Kot, Bull. Polon. Acad. Sci.: Tech. Sci. 59, 343 (2011).

14. A. D. Pogrebnjak, S. Bratushka, V. I. Boyko, I. V. Shamanin, and Y. V. Tsvintarnaya, Nucl. Instrum. Meth. Phys. Res. B 145, 373 (1998).

15. A. Gilewicz and B. Warcholinski, Tribol. Int. 80, 34 (2014).

16. R. A. Koshy, M. E. Graham, and L. D. Marks, Surf. Coat. Technol. 202, 1123 (2007).

17. V. Ivashchenko, S. Veprek, A. Pogrebnjak, and B. Postolnyi, Sci. Technol. Adv. Mater. 15, 25007 (2014).

18. A. Komarov, F. Komarov, P. Żukowski, C. Karwat, and A. Kamarou, Vacuum 63, 495 (2001).
19. A. D. Pogrebnyak, O. M. Ivasishin, and V. M. Beresnev, Usp. Fiz. Met. 17, 1 (2016).

20. A. D. Pogrebnjak, D. Eyidi, G. Abadias, O. V. Bondar, V. M. Beresnev, and O. V. Sobol, Int. J. Refract. Met. Hard Mater. 48, 222 (2015).

21. L. S. Metlov, M. M. Myshlyaev, A. V. Khomenko, and Ya. A. Lyashenko, Tech. Phys. Lett. 38, 972 (2012).

22. S. Veprek, M. G. J. Veprek-Heijman, P. Karvankova, and J. Prochazka, Thin Solid Films 476, 1 (2005).

23. A. D. Pogrebnjak, G. Abadias, O. V. Bondar, B. O. Postolnyi, M. O. Lisovenko, O. V. Kyrychenko, A. A. Andreev, V. M. Beresnev, D. A. Kolesnikov, and M. Opielak, Acta Phys. Polon. A 125, 1280 (2014).

Translated by G. Dedkov

Influence of the Bilayer Thickness of Nanostructured Multilayer MoN/CrN Coating on Its Microstructure, Hardness, and Elemental Composition [Текст] / A .D. Pogrebnyak, O.V. Bondar, B. Zhollybekov [та ін.] // Physics of the Solid State. — 2017. — №59(9). — C. 1798-1802. 